\title{
Hilft beim postthrombotischen Syndrom ein Gehtraining?
}

\begin{abstract}
Nach einer tiefen Beinvenenthrombose entwickelt sich bei bis zu $50 \%$ der Patienten ein chronisches postthrombotisches Syndrom (PTS). Ob ein Gehtraining die Symptome und die Lebensqualität dieser Patienten verbessern kann, wurde nun untersucht.
\end{abstract}

- Um die Machbarkeit eines Gehtrainings zur Behandlung des PTS zu prüfen, wurden 43 Patienten mit einer einseitigen tiefen Venenthrombose zu einem sechsmonatigen strukturierten und supervidierten Gehtrainingsprogramm randomisiert, das Elemente zur Kräftigung und Dehnung sowie ein aerobes Training enthielt. Dieses Training fand innerhalb der ersten beiden Wochen dreimal wöchentlich, während der dritten Woche zweimal wöchentlich und danach einmal wöchentlich statt.

Die Kontrollgruppe sah einen standardisierten Diavortrag über das PTS und wurde nach einem, zwei, vier und fünf Monaten telefonisch kontaktiert und zum Gesamtzustand und zu Beinsymptomen befragt. Zudem wurde die venenspezifische Lebensqualität mit dem VEINES-Fragebogen erfragt und der

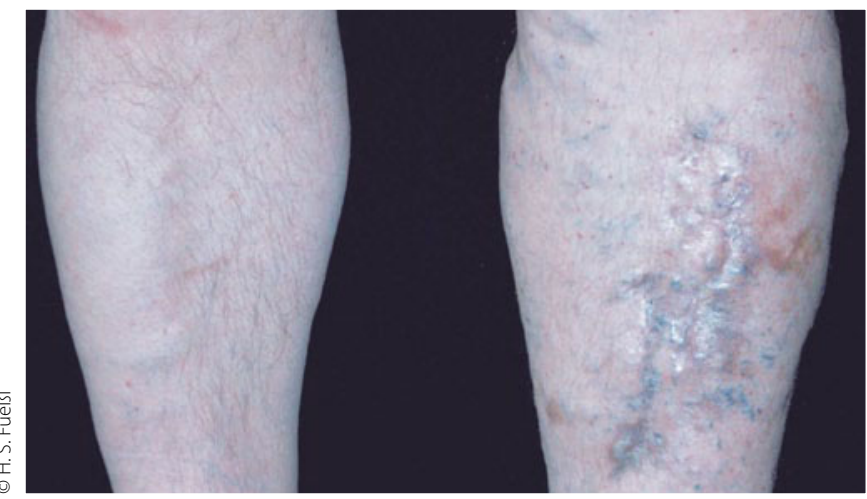

Etwa jeder zweite Patient mit tiefer Beinvenenthrombose entwickelt ein postthrombotisches Syndrom.
Schweregrad des PTS mithilfe der Villalta-Skala bestimmt. Zum Schluss wurden die Kraft, Flexibilität und Übungskapazität auf dem Laufband untersucht.

Nach drei Monaten hatten drei Patienten aus der Trainingsgruppe das Trainingsprogramm aufgegeben. Nach sechs Monaten zeigten sich in der Trainingsgruppe signifikante Verbesserungen im VEINES-Fragebogen (durchschnittliche Veränderung: 6,0 versus 1,4; 95\%-Konfidenzintervall: $0,54-8,7 ; p=0,027$ ).

In dieser Pilotstudie konnten keine Veränderungen im Villalta-Score gefunden werden. Jedoch war das Trainingsprogramm mit einer deutlichen Verbesserung der Kraft und Flexibilität der unteren Extremitäten verbunden.

\section{Kommentar}

Die Autoren schlussfolgerten, dass ein strukturiertes Training die Folgen des PTS verbessern kann. Jedoch sollten diese Ergebnisse in einer größer angelegten Studie überprüft werden. Obwohl die Rate derjenigen, die das Training aufgaben, deutlich niedriger war als man das aus der konservativen Therapie von Patienten mit peripherer arterieller Verschlusskrankheit kennt, wäre es interessant zu sehen, ob ein weniger aufwendiges Training einen ähnlichen Effekt bei Patienten mit tiefer Beinvenenthrombose erbringen kann.

N. DIEHM =

\section{- S. R. Kahn et al.}

Six-month exercise training program to treat post-thrombotic syndrome: a randomized controlled two-centre trial. CMAJ 183 (2011) 37-44

\section{Melatonin bei Delirium?}

\section{Ein Delirium ist häufig bei Senioren, die hospitalisiert werden müssen, und es ist zudem mit erheblichen Risiken verbunden. Eine effektive Prävention wäre daher willkommen.}

— Kanadische Geriater haben 145 ältere Patienten, die wegen verschiedener Gebrechen hospitalisiert wurden, in zwei Gruppen randomisiert. Zusätzlich zur jeweils indizierten Standardtherapie erhielt eine Gruppe 14 Tage lang täglich $0,5 \mathrm{mg}$ Melatonin, während der Kontrollgruppe
Placebos verabreicht wurden. In der Verumgruppe litten $12 \%$ und in der Kontrollgruppe $31 \%$ an einem Delirium. Die Autoren meinen daher, dass niedrig dosiertes Melatonin ein wirksames Mittel sei, Delirium bei älteren, hospitalisierten Patienten zu verhindern.

$$
\text { Kommentar }
$$

Die hier vorgelegten Daten sind beeindruckend. Die Autoren postulieren, dass die Melatoningabe den Metabolismus von Tryptophan und Serotonin verlangsamt, was die
Wirksamkeit bei dieser Indikation zumindest teilweise erklären könnte. Melatonin ist nicht teuer und hat kaum Nebenwirkungen. Somit ist es eine äußerst attraktive Innovation in der Deliriumsprophylaxe. Dennoch, so würde ich meinen, brauchen wir eine unabhängige Bestätigung dieser Daten, ehe wir dieses Konzept in die klinische Routine übernehmen können.

E. ERNST :

\section{- T. Al-Aama et al.}

Melatonin decreases delirium in elderly patients: a randomized, placebo-controlled trial. Int J Geriatr Psychiatry 26 (2011) 687-694 